\title{
Clinical Practice of Two Measurements of Home Blood Pressure on Each Occasion in Patients with Chronic Kidney Disease
}

\author{
Tomonari Okada Toshikazu Wada Yume Nagaoka Yoshihiko Kanno \\ Department of Nephrology, Tokyo Medical University, Tokyo, Japan
}

\section{Key Words}

Home blood pressure - Self-measurement of blood pressure - Blood pressure variability ·

Chronic kidney disease $\cdot$ Hypertension

\begin{abstract}
Background/Aims: Although several guidelines propose two or three measurements of home blood pressure (HBP) on each occasion, the actual status of multiple measurements is not clear in the practical management of hypertension. We surveyed the details regarding two measurements of HBP in patients with chronic kidney disease (CKD). Methods: HBP was measured twice every morning and evening over 7 consecutive days in 175 CKD patients. The distribution of the differences between two BP values (2nd - 1st BP) and their association with BP parameters were evaluated. Results: The 2 nd - 1st morning systolic BP (SBP) and diastolic $B P$ (DBP) differences were $-2.3 \pm 4.1$ and $-0.4 \pm 2.6 \mathrm{~mm} \mathrm{Hg}$, respectively. The proportion of 2nd - 1st morning SBP differences $>0 \mathrm{~mm} \mathrm{Hg}$ was $31.7 \%$ in a total of 1,195 measurements. Eighty patients (45.7\%) had days with a difference $\leq-5 \mathrm{~mm} \mathrm{Hg}$ and days with a difference $\geq 5$ $\mathrm{mm} \mathrm{Hg}$ in morning SBP during 7 days. The multivariate regression analysis of the SD values of 2 nd - 1st morning SBP as a dependent variable showed that the SD value of the 1st morning SBP $(\beta=0.65, p<0.001)$ was a significant determinant. Conclusion: Although the 2nd SBP was $2-3 \mathrm{~mm} \mathrm{Hg}$ lower than the 1 st SBP in the population as a whole, various differences were found for each subject during 7 days. 2nd - 1st BP variability might be associated with dayby-day 1st BP variability.

(C) 2015 S. Karger AG, Basel
\end{abstract}

\section{Introduction}

The measurement of home blood pressure (HBP) is popular in the management of hypertension in everyday practice. HBP has great advantages over clinical BP, including its reproducibility, exclusion of the white-coat effect, an association with target organ damage, and a 


\section{CardioRenal Medicine}

\begin{tabular}{l|l}
\hline Cardiorenal Med 2016;6:8-15 \\
\hline DOI: 10.1159/000438464 & $\begin{array}{l}\text { @ 2015 S. Karger AG, Basel } \\
\text { www.karger.com/crm }\end{array}$ \\
\hline
\end{tabular}

Okada et al.: Clinical Practice of Two Measurements of Home Blood Pressure on Each Occasion in Patients with Chronic Kidney Disease

better prognostic value [1-5]. Many BP data can easily be obtained during the course of a day and over a long period by self-measurement at home. Therefore, it is necessary to standardize the conditions of measurement. Among the several issues regarding these conditions, how many times measurements should be taken on each occasion has not yet been resolved [6]. The American and Japanese guidelines propose two measurements on each occasion [7, 8]. The European guideline proposes three measurements on each occasion [9]. Many previous studies have shown that the BP of the second measurement was lower than that of the first measurement [10-16]. However, it is not clear whether this tendency is a consistent finding for each subject over a long period. In addition, the superiority of the prognostic value of two measurements is not consistent.

Although it is extremely important to manage BP control based on HBP in the medical treatment of chronic kidney disease (CKD) patients, the details regarding the use of two measurements of HBP in these patients are not clear in clinical practice. The objective of this study is to clarify the actual state of two measurements of HBP on each occasion in CKD patients.

\section{Subjects and Methods}

\section{Patients}

We enrolled 175 patients treated at our department between May 2012 and February 2013 who met the eligibility criteria. As a first criterion for inclusion, CKD patients had to have been treated for $\geq 6$ months at our clinic. CKD was diagnosed either by the presence of overt proteinuria or by decreased kidney function according to an estimated glomerular filtration rate (eGFR) $<60 \mathrm{ml} / \mathrm{min} / 1.73 \mathrm{~m}^{2}$ [17]. As a second criterion, the patients' HBP measurements had to have already been obtained at regular intervals before participation in this study. We excluded those patients who started dialysis therapy or who had atrial fibrillation.

Of the 175 patients, 122 were men. The mean age at the time of HBP data collection was $69 \pm 11$ years (range 28-98). The underlying renal diseases were as follows: chronic glomerulonephritis ( $\mathrm{n}=63)$, nephrosclerosis $(n=57)$, diabetic nephropathy $(n=45)$, polycystic kidney disease $(n=5)$, tubulointerstitial nephritis $(n=2)$, and reflux nephropathy $(n=3)$. These diseases were diagnosed on the basis of the medical history and renal biopsy findings.

The mean baseline serum creatinine level was $2.7 \pm 2.0 \mathrm{mg} / \mathrm{dl}$ (range 0.57-10.70), and the mean eGFR was $28.7 \pm 19.7 \mathrm{ml} / \mathrm{min} / 1.73 \mathrm{~m}^{2}$ (range 4.2-81.5; CKD stages 2, 3, 4, and 5: $\mathrm{n}=18,45$, 60, and 52, respectively), calculated by the following formula for Japanese patients: $194 \times \mathrm{Cr}^{-1.094} \times$ age $^{-0.287}(\times 0.739$ for women) [18]. The mean creatinine clearance rate was $37.9 \pm 29.6 \mathrm{ml} / \mathrm{min}$ (range $5.1-174.0 ; \mathrm{n}=150$ ), the mean urinary protein excretion rate was $1.28 \pm 1.53 \mathrm{~g} /$ day (range $0-9.1 ; \mathrm{n}=150$ ), and the mean sodium excretion rate was $144 \pm 65 \mathrm{mEq} /$ day $(\mathrm{n}=150)$. The mean hemoglobin level was $12.2 \pm 1.7 \mathrm{~g} / \mathrm{dl}$, and the mean serum uric acid level was $7.2 \pm 1.6 \mathrm{mg} / \mathrm{dl}$. The mean body mass index was $24.1 \pm 3.9$.

\section{HBP Measurements and Data Collection}

HBP was measured according to the guidelines for HBP measurement of the Japanese Society of Hypertension (JSH) [19]. BP was measured using oscillometric arm devices in a seated position after at least 1 min of rest. It was measured twice, with an interval of 1 min between measurements - in the morning within $1 \mathrm{~h}$ after waking before dosing, and in the evening just before bedtime. The values of the two measurements were recorded at each of these time points. The patients recorded 28 BP readings as described above over 7 days preceding their clinic visit. The first measurements (1st BP) and the second measurements (2nd BP) of the morning and evening BP values taken over 7 days were averaged for each patient. The differences between the 1st BP and the 2nd BP (2nd - 1st BP) were obtained and averaged for each patient. In 56 patients, HBP data were recorded with the same method after $1.6 \pm 0.8$ months (range 1-4) without changes in antihypertensive drugs.

This study was approved by the Ethics Committee of the Tokyo Medical University (No. 2924), and all patients provided written informed consent to use their clinical data in this study.

\section{Treatment at the Time of Data Collection}

We treated the patients according to the guidelines of the JSH [19]. Antihypertensive drugs were selected according to the guidelines of the JSH as well [20]. The numbers of antihypertensive drugs taken by the 
Table 1. BP and heart rate parameters

\begin{tabular}{lcccc}
\hline & 1st & 2nd & 2nd - 1st & p \\
\hline Morning SBP, mm Hg & $135.7 \pm 14.9$ & $133.4 \pm 14.5$ & $-2.3 \pm 4.1$ & $<0.001$ \\
SD of morning SBP, mm Hg & $7.3 \pm 3.5$ & $7.2 \pm 3.5$ & & 0.62 \\
Morning DBP, mm Hg & $76.5 \pm 10.3$ & $76.1 \pm 10.0$ & $-0.4 \pm 2.6$ & 0.005 \\
SD of morning DBP, mm Hg & $4.7 \pm 2.1$ & $4.6 \pm 2.3$ & & 0.27 \\
Evening SBP, mm Hg & $130.1 \pm 14.6$ & $127.9 \pm 14.7$ & $-2.3 \pm 4.4$ & $<0.001$ \\
SD of evening SBP, mm Hg & $8.6 \pm 4.8$ & $8.1 \pm 4.3$ & & 0.28 \\
Evening DBP, mm Hg & $71.6 \pm 10.2$ & $71.5 \pm 9.9$ & $-0.2 \pm 2.5$ & 0.06 \\
SD of evening DBP, mm Hg & $5.3 \pm 2.5$ & $5.2 \pm 2.6$ & & 0.21 \\
Morning heart rate, bpm & $69.7 \pm 10.5$ & $68.6 \pm 10.0$ & & $<0.001$ \\
Evening heart rate, bpm & $72.1 \pm 10.0$ & $71.0 \pm 10.0$ & & $<0.001$ \\
\hline
\end{tabular}

subjects at the time of HBP data collection were as follows: $0(n=14), 1(n=39), 2(n=58), 3(n=43)$, and $\geq 4(n=21)$. The types of antihypertensive drugs taken by the subjects were as follows: angiotensin receptor blockers $(n=130)$, calcium channel blockers $(n=127), \alpha_{1}$-blockers $(n=36)$, angiotensin-converting enzyme inhibitors $(n=27), \alpha \beta$-blockers $(n=21)$, and $\beta_{1}$-blockers $(n=12)$. Furosemide, thiazide, and an aldosterone blocker were given to 34,18 , and 5 patients, respectively.

\section{Statistical Analysis}

Data are expressed as means \pm SD. p values $<0.05$ were considered to represent statistically significant differences. Changes in BP parameters between measurements were analyzed by the Wilcoxon signed-rank test. The values of $2 \mathrm{nd}-1$ st BP at the first time point and those at the second time point were analyzed by the Mann-Whitney test. Correlations between BP variables were analyzed by the Pearson correlation coefficient. Multiple regression analysis was performed to identify the independent variables associated with 2nd - 1st BP. Statistical analyses were performed using SPSS version 21 (IBM, Tokyo, Japan).

\section{Results}

\section{BP Parameters}

Table 1 shows the BP and heart rate parameters. The 2 nd -1 st morning systolic/diastolic BP (SBP/DBP) and 2nd - 1st evening SBP/DBP differences were $-2.3 \pm 4.1 /-0.4 \pm 2.6$ and $-2.3 \pm 4.4 /-0.2 \pm 2.5 \mathrm{~mm} \mathrm{Hg}$, respectively. The 2nd morning SBP, morning DBP, and evening SBP values were significantly lower than their respective 1 st BP measurements. No significant changes were found between the SD values of 1st BP and those of 2nd BP.

\section{Distributions of 2 nd - 1st SBP}

Table 2 shows the distribution of the numbers of patients and measurements according to 2 nd -1 st SBP. The distribution of the numbers of patients was based on the average values of 2 nd - 1st SBP during 7 days. The distribution of the numbers of measurements was based on each value of 2 nd - 1st SBP in the whole patient population during 7 days. The greatest proportion of 2nd - 1st SBP differences lay within the range of 0 to $-4.9 \mathrm{~mm} \mathrm{Hg}$ (patients: morning 52.0\%, evening 45.1\%; measurements: morning 34.6\%, evening 30.4\%). The proportion of patients whose $2 \mathrm{nd}-1$ st SBP differences were $>0 \mathrm{~mm} \mathrm{Hg}$ was $26.3 \%$ in the morning and $30.9 \%$ in the evening, respectively. The proportion of measurements $>0 \mathrm{~mm} \mathrm{Hg}$ for 2 nd - 1st SBP was $31.7 \%$ in the morning and $34.2 \%$ in the evening, respectively.

Table 3 shows the distribution of patient numbers according to 2nd - 1st SBP during 7 days; 80 patients (45.7\%) had days with a difference $\leq-5 \mathrm{~mm} \mathrm{Hg}$ and days with a difference $\geq 5 \mathrm{~mm} \mathrm{Hg}$ in 2nd - 1st morning SBP. 
Okada et al.: Clinical Practice of Two Measurements of Home Blood Pressure on Each Occasion in Patients with Chronic Kidney Disease

Table 2. Distribution of the differences between 1st and 2nd measurements of SBP (2nd - 1st SBP)

\begin{tabular}{lclll}
\hline & $\begin{array}{l}\text { Morning } \\
(\mathrm{n}=175 \\
\text { patients })\end{array}$ & $\begin{array}{l}\text { Evening } \\
(\mathrm{n}=175 \\
\text { patients })\end{array}$ & $\begin{array}{l}\text { Morning } \\
(\mathrm{n}=1,195 \\
\text { measurements })\end{array}$ & $\begin{array}{l}\text { Evening } \\
(\mathrm{n}=1,189 \\
\text { measurements })\end{array}$ \\
\hline$\leq-10 \mathrm{~mm} \mathrm{Hg}$ & $6(3.4 \%)$ & $11(6.3 \%)$ & $168(14.1 \%)$ & $186(15.6 \%)$ \\
-5 to $-9.9 \mathrm{~mm} \mathrm{Hg}$ & $32(18.3 \%)$ & $31(17.7 \%)$ & $234(19.6 \%)$ & $231(19.3 \%)$ \\
0 to $-4.9 \mathrm{~mm} \mathrm{Hg}$ & $91(52.0 \%)$ & $79(45.1 \%)$ & $414(34.6 \%)$ & $363(30.4 \%)$ \\
0.1 to $4.9 \mathrm{~mm} \mathrm{Hg}$ & $40(22.8 \%)$ & $43(24.6 \%)$ & $209(17.5 \%)$ & $219(18.3 \%)$ \\
5 to $9.9 \mathrm{~mm} \mathrm{Hg}$ & $5(2.9 \%)$ & $11(6.3 \%)$ & $99(8.3 \%)$ & $115(9.6 \%)$ \\
$\geq 10 \mathrm{~mm} \mathrm{Hg}$ & $1(0.6 \%)$ & $0(0.0 \%)$ & $71(5.9 \%)$ & $75(6.3 \%)$ \\
\hline
\end{tabular}

Table 3. Distribution of patient numbers according to 2 nd - 1st SBP during 7 days

2nd - 1st morning SBP

Patients who had days with a difference $\leq-5 \mathrm{~mm} \mathrm{Hg}$

$69(39.4 \%)^{\mathrm{a}}$

Patients who had days with a difference $\leq-5 \mathrm{~mm} \mathrm{Hg}$ and days with a difference $\geq 5 \mathrm{~mm} \mathrm{Hg} \quad 80(45.7 \%)^{\mathrm{b}}$

Patients who had days with a difference $\geq 5 \mathrm{~mm} \mathrm{Hg}$

Patients who had days with a difference within the range of $>-5$ and $<5 \mathrm{~mm} \mathrm{Hg}$

$15(8.6 \%)^{\mathrm{c}}$

$11(6.3 \%)^{\mathrm{d}}$

2nd - 1st evening SBP

Patients who had days with a difference $\leq-5 \mathrm{~mm} \mathrm{Hg}$

$65(37.1 \%)$

Patients who had days with a difference $\leq-5 \mathrm{~mm} \mathrm{Hg}$ and days with a difference $\geq 5 \mathrm{~mm} \mathrm{Hg} \quad 79(45.1 \%)$

Patients who had days with a difference $\geq 5 \mathrm{~mm} \mathrm{Hg}$

$18(10.3 \%)$

Patients who had days with a difference within the range of $>-5$ and $<5 \mathrm{~mm} \mathrm{Hg}$

$13(7.5 \%)$

${ }^{a} 44$ patients had days with a difference $\leq-5 \mathrm{~mm} \mathrm{Hg}$ in the evening. ${ }^{\mathrm{b}} 55$ patients had days with a difference $\leq-5 \mathrm{~mm} \mathrm{Hg}$ and days with a difference $\geq 5 \mathrm{~mm} \mathrm{Hg}$ in the evening. ${ }^{c} 10$ patients had days with a difference $\leq-5$ $\mathrm{mm} \mathrm{Hg}$ in the evening. ${ }^{\mathrm{d}} 9$ patients had days with a difference within the range of $>-5$ and $<5 \mathrm{~mm} \mathrm{Hg}$ in the evening.

\section{Correlations between 2nd - 1st SBP and Clinical Parameters}

There was a weak but significant correlation between 2nd - 1st morning SBP and 2nd 1 st evening SBP ( $r=0.30, \mathrm{p}<0.001)$. There was no significant difference between $2 \mathrm{nd}-1 \mathrm{st}$ morning SBP in males and that in females $(-2.2 \pm 4.3$ vs. $-2.7 \pm 3.6 \mathrm{~mm} \mathrm{Hg}, \mathrm{p}=0.73)$. There were no significant differences in 2nd - 1st morning SBP between the patients with chronic glomerulonephritis, those with nephrosclerosis, and those with diabetic nephropathy (data not shown). There were no significant correlations between age, eGFR, the number of antihypertensive drugs, and 2nd - 1st morning SBP (data not shown).

The multivariate regression analysis of 2 nd -1 st morning SBP as a dependent variable showed that 1 st morning SBP $(\beta=-0.27, p=0.01)$ was the only significant determinant, whereas age, sex, eGFR, body mass index, diabetes mellitus, and the number of antihypertensive drugs were not $\left(\mathrm{R}^{2}=0.07\right)$.

\section{Variability of 2nd - 1st BP}

The mean SD values of 2 nd -1 st morning and evening BP were $6.2 \pm 3.4 / 4.3 \pm 2.4$ and $6.5 \pm 3.6 / 4.6 \pm 2.9 \mathrm{~mm} \mathrm{Hg}$, respectively. The mean SD value of $2 \mathrm{nd}-1$ st morning SBP was not significantly associated with 1st morning SBP and 2nd - 1st morning SBP $(r=-0.12$ and $r=$ $-0.05, p=0.09$ and $p=0.55$ ). However, it was significantly associated with the mean SD value of 1st morning SBP ( $r=0.64, p<0.001)$ (fig. 1$)$. The mean SD value of 2 nd -1 st evening SBP 


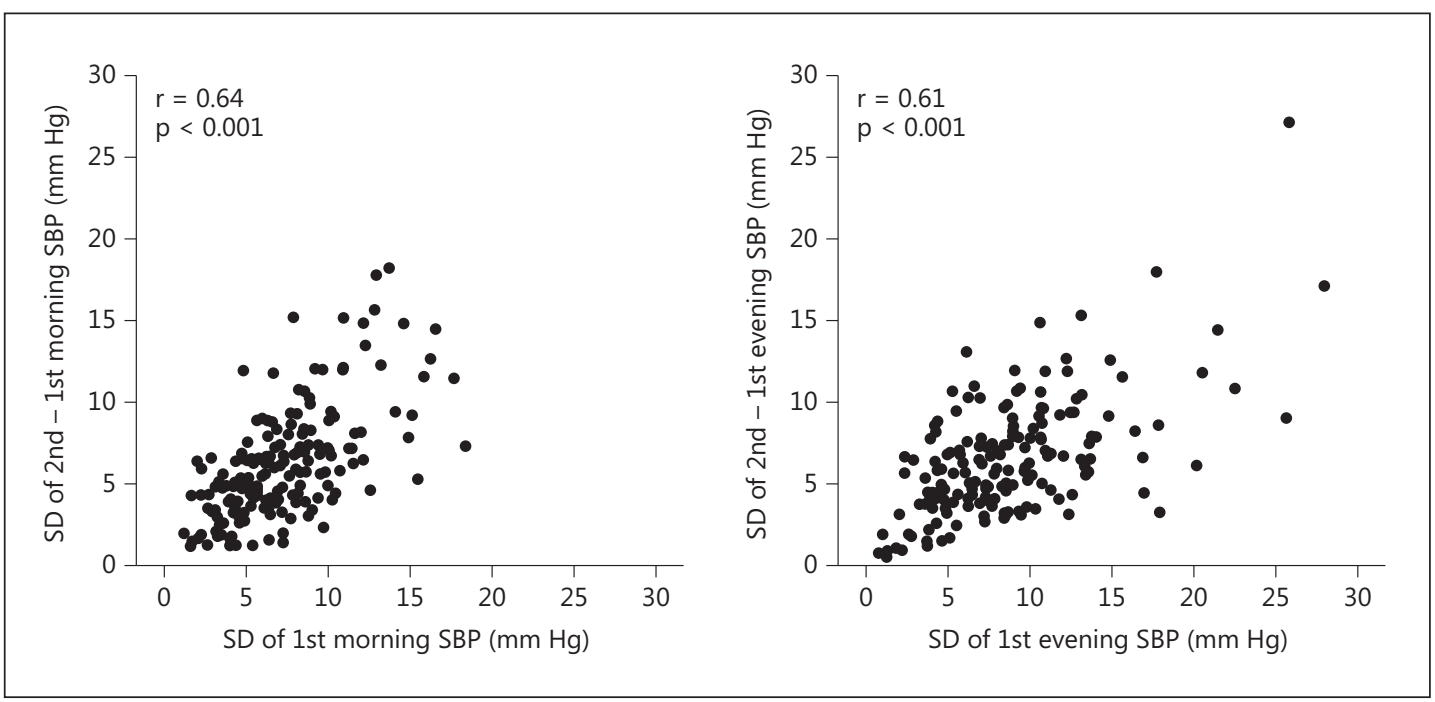

Fig. 1. Correlations between SD values of 1 st SBP and SD values of 2 nd - 1st SBP during 7 days.

had similar results. The multivariate regression analysis of the mean SD value of 2 nd -1 st morning SBP as a dependent variable showed that the mean SD value of 1st morning SBP $(\beta=0.65, p<0.001)$ was a significant determinant and female sex was a borderline significant determinant $(\beta=0.12, p=0.05)$, whereas age, eGFR, body mass index, diabetes mellitus, and the number of antihypertensive drugs were not $\left(R^{2}=0.66\right)$.

\section{Reproducibility of 2 nd - 1st BP}

In the 56 patients who recorded their HBP data by the same method after $1.6 \pm 0.8$ months, there were no significant differences between BP values at the first time point and those at the second time point $(137.3 \pm 16.4 / 77.5 \pm 11.3 \mathrm{~mm} \mathrm{Hg}$ in the morning at the first and $138.7 \pm 17.6 / 78.1 \pm 10.8 \mathrm{~mm} \mathrm{Hg}$ in the morning at the second time point; $131.3 \pm 16.8$ / $72.7 \pm 10.7 \mathrm{~mm} \mathrm{Hg}$ in the evening at the first and $132.8 \pm 16.3 / 73.2 \pm 10.6 \mathrm{~mm} \mathrm{Hg}$ in the evening at the second time point). There was no significant difference between 2 nd -1 st morning BP at the first time point and those at the second time point $(-2.9 \pm 4.3 /-0.6 \pm 2.6$ and $-2.2 \pm 4.1 /-0.5 \pm 2.5 \mathrm{~mm}$ Hg; SBP: $\mathrm{p}=0.41$; DBP: $\mathrm{p}=0.59$ ). The 2 nd -1 st evening BP showed similar results (data not shown). There was a significant correlation between $2 \mathrm{nd}-$ 1 st SBP at the first time point and those at the second time point (morning: $r=0.35, p=0.008$; evening: $r=0.46, p<0.001$ ).

\section{Discussion}

The present study showed the various differences in 2nd - 1st BP for each subject during 7 days. Although the 2nd SBP was 2-3 mm Hg lower than the 1st SBP in the population as a whole, about $30 \%$ of the patients had $2 \mathrm{nd}-1 \mathrm{st}$ SBP $>0 \mathrm{~mm}$ Hg. The results of our study agree with those of the previous studies, in which the mean values of 2 nd measurements were 2-3/1-2 mm Hg lower than the mean values of 1st measurements on each occasion [10-16]. However, the details regarding the differences between the two measurements were not clarified in these previous studies. One of these studies showed that $30-40 \%$ of healthy individuals had higher 2 nd measurement than 1st measurement values [11]. In the Home Blood 
Pressure Measurement with Olmesartan Naive Patients to Establish Standard Target Blood Pressure (HONEST) study, the greatest proportion of 2nd - 1st morning SBP differences lay within the range of 0 to $-4.9 \mathrm{~mm} \mathrm{Hg} \mathrm{(43.1 \% )} \mathrm{in} \mathrm{22,162} \mathrm{patients} \mathrm{with} \mathrm{essential} \mathrm{hypertension}$ [16]. The proportion of patients whose $2 \mathrm{nd}-1$ st morning SBP differences were $>0 \mathrm{~mm} \mathrm{Hg}$ was $26.3 \%$. Only $6.8 \%$ of the included patients had renal diseases, and the eGFR was $72 \pm 20$ $\mathrm{ml} / \mathrm{min} / 1.73 \mathrm{~m}^{2}$. Although this study obtained averaged BP values from only 2 days, the distribution of patients according to BP changes was very similar to that of the present study. In the present study, no significant association between eGFR and 2nd - 1st morning SBP was found in the multivariate regression analysis. Thus, the presence of CKD might not affect the changes of BP.

One previous study showed the superiority of the 2nd BP measurement for prognosis or an association with target organ damage [15]. On the other hand, some studies have shown similar values between two measurements $[13,14]$. Other studies have shown the prognostic value of one BP measurement on each occasion [4, 5, 21]. From these studies, the prognostic value of two measurements cannot be consistently derived. Further studies are necessary to clarify whether continuous double measurement is more beneficial than continuous single measurement with regard to the management of BP control.

In the present study, the 1st SBP was the only significant determinant of 2nd - 1st BP in the multivariate regression analysis. We could not identify the factors related to the changes of BP. However, many behavioral and physiological factors associated with short-term BP variability might influence changes of BP [22]. Increased sympathetic activity, decreased baroreflex activity, and arterial stiffness might be possible factors involved in the changes of $\mathrm{BP}$ in multiple measurements in the same way as the short-term BP variability assessed by ambulatory BP monitoring. Further studies are necessary to identify the factors related to changes of BP in multiple measurements.

The SD values of 2 nd - 1st BP might represent as an index of short-term BP variability. Data regarding the variability of 2nd - 1st BP are scarce. In the Finn-Home Study, old age, female sex, history of cardiovascular disease, and high HBP were independent determinants of greater variability in 2nd - 1st measurements of HBP [23]. In the present study, female sex was a borderline significant determinant of SD values of 2 nd - 1st morning SBP in the multivariate regression analysis. In addition, the SD values of 2 nd - 1st SBP were significantly associated with the SD values of 1st SBP both in the morning and in the evening. To the best of our knowledge, there are as yet no studies regarding the relation between these variabilities. From these results, variability in 2 nd - 1st measurements might be significantly associated with day-by-day variability. In the Finn-Home Study, variability in diastolic 2 nd -1 st measurement of BP predicted cardiovascular events as well as morning day-by-day diastolic $\mathrm{BP}$ variability. Therefore, variability in $2 \mathrm{nd}-1$ st measurements might have a clinical significance similar to that of day-by-day variability [23]. Further studies are necessary to clarify the clinical significance of the relation between these variabilities.

The present study assessed the reproducibility of 2 nd - 1st BP. One previous study has shown the agreement of the 1 st measurement and the 2 nd measurement between separated periods in healthy volunteers [12]. It is not clear whether this tendency would be applicable to treated hypertensive patients. Although the correlation was weak in the present study, some populations might have characteristics which entail a consistent change of BP during two measurements.

This study has some limitations. First, the study cohort was small, possibly limiting the statistical power. Also, only patients who continued HBP measurements regularly were included in the study; thus, a bias in the selection of the study population cannot be completely ruled out. Second, although BP was measured twice with an interval of 1 min between measurements, the condition during the interval between measurements was not uniform. 
Okada et al:: Clinical Practice of Two Measurements of Home Blood Pressure on Each

In conclusion, although the 2 nd BP has lower values than the 1st BP in the population as a whole, various differences between two measurements were found for each subject during 7 days in patients with CKD. 2nd - 1st BP variability was significantly associated with day-byday variability. Further studies are necessary to clarify the factors affecting the BP change between two measurements, and to clarify the significance of two measurements of HBP on each occasion in clinical practice.

\section{Acknowledgement}

The authors are indebted to Assoc. Prof. Edward F. Barroga, Senior Editor of the Department of International Medical Communications of Tokyo Medical University, for the editorial review of the manuscript.

\section{Disclosure Statement}

The authors report no conflicts of interest and no funding for the work.

\section{References}

1 Sakuma M, Imai Y, Nagai K, Watanabe N, Sakuma H, Minami N, et al: Reproducibility of home blood pressure measurements over a 1-year period. Am J Hypertens 1997;10:798-803.

2 Hozawa A, Ohkubo T, Kikuya M, Yamaguchi J, Ohmori K, Fujiwara T, et al: Blood pressure control assessed by home, ambulatory and conventional blood pressure measurements in the Japanese general population: the Ohasama study. Hypertens Res 2002;25:57-63.

3 Stergiou GS, Argyraki KK, Moyssakis I, Mastorantonakis SE, Achimastos AD, Karamanos VG, et al: Home blood pressure is as reliable as ambulatory blood pressure in predicting target-organ damage in hypertension. Am J Hypertens 2007;20:616-621.

4 Ohkubo T, Imai Y, Tsuji I, Nagai K, Kato J, Kikuchi N, et al: Home blood pressure measurement has a stronger predictive power for mortality than does screening blood pressure measurement: a population-based observation in Ohasama, Japan. J Hypertens 1998;16:971-975.

5 Sega R, Facchetti R, Bombelli M, Cesana G, Corrao G, Grassi G, et al: Prognostic value of ambulatory and home blood pressures compared with office blood pressure in the general population: follow-up results from the Pressioni Arteriose Monitorate e Loro Associazioni (PAMELA) study. Circulation 2005;111:1777-1832.

6 Imai Y, Obara T, Ohkubo T: How many times should we ask subjects to measure blood pressure at home on each occasion? J Hypertens 2007;25:1987-1991.

7 Pickering TG, Miller NH, Ogedegbe G, Krakoff LR, Artinian NT, Goff D; American Heart Association; American Society of Hypertension; Preventive Cardiovascular Nurses Association: Call to action on use and reimbursement for home blood pressure monitoring: a joint scientific statement from the American Heart Association, American Society of Hypertension, and Preventive Cardiovascular Nurses Association. Hypertension 2008;52:10-29.

8 Shimamoto K, Ando K, Fujita T, Hasebe N, Higaki J, Horiuchi M, et al: The Japanese Society of Hypertension Guidelines for the Management of Hypertension (JSH 2014). Chapter 2. Measurement and clinical evaluation of blood pressure. Hypertens Res 2014;37:266-278.

9 Parati G, Stergiou GS, Asmar R, Bilo G, de Leeuw P, Imai Y, et al: European Society of Hypertension guidelines for blood pressure monitoring at home: a summary report of the Second International Consensus Conference on Home Blood Pressure Monitoring. J Hypertens 2008;26:1505-1526.

10 Stergiou GS, Skeva II, Zourbaki AS, Mountokalakis TD: Self-monitoring of blood pressure at home: how many measurements are needed? J Hypertens 1998;16:725-731.

11 Kawabe H, Saito I, Saruta T: Influence of repeated measurement on one occasion, on successive days, and on workdays on home blood pressure values. Clin Exp Hypertens 2005;27:215-222.

12 Kawabe H, Saito I: Which measurement of home blood pressure should be used for clinical evaluation when multiple measurements are made? J Hypertens 2007;25:1369-1374.

13 Johansson JK, Niiranen TJ, Puukka PJ, Jula AM: Optimal schedule for home blood pressure monitoring based on a clinical approach. J Hypertens 2010;28:259-264.

14 Stergiou GS, Nasothimiou EG, Kalogeropoulos PG, Pantazis N, Baibas NM: The optimal home blood pressure monitoring schedule based on the Didima outcome study. J Hum Hypertens 2010;24:158-164.

15 Niiranen TJ, Johansson JK, Reunanen A, Jula AM: Optimal schedule for home blood pressure measurement based on prognostic data: the Finn-Home Study. Hypertension 2011;57:1081-1086. 
16 Saito I, Kario K, Kushiro T, Teramukai S, Zenimura N, Hiramatsu K, et al: Rationale, study design, baseline characteristics and blood pressure at 16 weeks in the HONEST Study. Hypertens Res 2013;36:177-182.

17 Japanese Society of Nephrology: Clinical Practice Guidebook for Diagnosis and Treatment of Chronic Kidney Disease. Chapter 2. Definition and classification of CKD. Clin Exp Nephrol 2009;13:196.

18 Japanese Society of Nephrology: Clinical Practice Guidebook for Diagnosis and Treatment of Chronic Kidney Disease. Chapter 9. Evaluation method for kidney function and urinary findings. Clin Exp Nephrol 2009;13: 209-211.

19 Ogihara T, Kikuchi K, Matsuoka H, Fujita T, Higaki J, Horiuchi M, et al: The Japanese Society of Hypertension Guidelines for the Management of Hypertension (JSH 2009). Chapter 2. Measurement and clinical evaluation of blood pressure. Hypertens Res 2009;32:11-23.

20 Ogihara T, Kikuchi K, Matsuoka H, Fujita T, Higaki J, Horiuchi M, et al: The Japanese Society of Hypertension Guidelines for the Management of Hypertension (JSH 2009). Chapter 5. Treatment with antihypertensive drugs. Hypertens Res 2009;32:33-39.

21 Okada T, Nakao T, Matsumoto H, Nagaoka Y: Value of morning home blood pressure as a predictor of decline in renal function in patients with chronic kidney disease. Am J Nephrol 2008;28:982-989.

22 Mancia G: Short- and long-term blood pressure variability: present and future. Hypertension 2012;60:512517.

23 Johansson JK, Niiranen TJ, Puukka PJ, Jula AM: Factors affecting the variability of home-measured blood pressure and heart rate: the Finn-Home Study. J Hypertens 2010;28:1836-1845. 